\title{
Notre revue Obésité en première ligne des événements scientifiques！
}

\section{J. Dargent $\cdot$ S. Czernichow}

C Springer-Verlag France 2012

Ce numéro de rentrée fait suite à celui qui était consacré principalement au congrès SOFFCO de Montpellier, qui a été, sous la présidence de David Nocca, un succès.

La réactivité de notre revue n'y a pas peu contribué, permettant aux participants de disposer de la totalité des abstracts dès le début de la manifestation.

Rappelons que bon nombre de ces abstracts vont faire l'objet d'une publication dans Obésité, de même que les mémoires du DIU de chirurgie de l'obésité.

Bon vent au congrès 2013 à Angers !
A une échelle plus modeste, nous produisons le résumé des faits marquants du second meeting "Noninva" de Lyon, qui a réuni, comme l'an dernier, les acteurs internationaux des "nouvelles technologies bariatriques".

Également dans ce numéro, une revue des problèmes obstétricaux après chirurgie bariatrique, un point sur la sleeve gastrectomie comme intervention de "reprise", sur l'anesthésie bariatrique, et sur l'activité physique. Enfin un article important sur l'Alliance Thérapeutique, version nord-américaine de notre "Éducation Thérapeutique", si importante aujourd'hui.

Bonne lecture et bonne rentrée à tous ! 